\title{
Development and Validation of a Nomogram to Assist Monitoring Nosocomial SARS-CoV-2 Infection of Hospitalized Patients
}

\author{
Chen Wang ${ }^{1, *}$, Chunyan Peng ${ }^{2, *}$, Leping Ning ${ }^{3 *}$, Xueping Qiu', Kaisong Wu ${ }^{4}, \mathrm{Na}^{\prime}$ Yang', Bingyu Jin', \\ Yue Zhao', Fang Zheng $\mathbb{D}^{\prime}$ \\ 'Center for Gene Diagnosis, Department of Laboratory Medicine, Zhongnan Hospital of Wuhan University, Wuhan, Hubei, People's Republic of China; \\ ${ }^{2}$ Department of Laboratory Medicine, Taihe hospital, Hubei University of Medicine, Shiyan, Hubei, People's Republic of China; ${ }^{3}$ Department of Laboratory \\ Medicine, The People's Hospital of Guangxi Zhuang Autonomous Region, Nanning, Guangxi, People's Republic of China; ${ }^{4}$ Department of Respiratory \\ Medicine, Zhongnan Hospital of Wuhan University, Wuhan, Hubei, People's Republic of China \\ *These authors contributed equally to this work
}

Correspondence: Fang Zheng, Center for Gene Diagnosis \& Department of Laboratory Medicine, Zhongnan Hospital of Wuhan University, Wuhan University, No. 169 Donghu Road, Wuchang District, Wuhan, 43007I, People's Republic of China, Tel +86-27-678I3233, Fax +86 27 678I3497, Email zhengfang@whu.edu.cn

Purpose: SARS-CoV-2 is extremely infectious, and the incidence of nosocomial infection is conceivably high. We aimed to develop and validate a nomogram to assist monitoring nosocomial SARS-CoV-2 infection in hospitalized patients.

Patients and Methods: There were 437 COVID-19 hospitalized cases and 420 negative inpatients enrolled from two hospitals in Hubei province, China. We compared the demographic and clinical characteristics of participants between the two groups. Then, LASSO regression and logistic regression were applied to build a nomogram for SARS-CoV-2 infection prediction in the development cohort. Our nomogram was assessed by area under the curve (AUC), calibration curve, decision curve (DCA) and clinical impact curve analysis (CICA).

Results: After LASSO regression filtration, eleven laboratory indicators were correlated with SARS-CoV-2 infection. Then, we integrated these features and constructed a nomogram, which showed a high AUC 0.863 (95\% CI: 0.834-0.892) in the development cohort with a sensitivity of $80.41 \%$ and specificity of $77.38 \%$ and 0.813 (95\% CI: $0.760-0.866)$ in validation cohort with a sensitivity of $82.98 \%$ and specificity of $70.43 \%$. The calibration plot displayed that the predicted outcomes were in good concordance with the actual observations. DCA and CICA further showed a larger clinical net benefit.

Conclusion: We constructed and validated a nomogram that integrated eleven laboratory indexes to assist monitoring of nosocomial SARS-CoV-2 infection in hospitalized patients. Our nomogram is remarkably informative for clinical practice, which will be helpful for preventing SARS-CoV-2 further transmission in hospital and avoiding nosocomial infection.

Keywords: COVID-19, nomogram, nosocomial SARS-CoV-2 infection, machine learning

\section{Introduction}

In December 2019, the outbreak of novel coronavirus disease 2019 (COVID-19) occurred in Wuhan, China, which is caused by the severe acute respiratory syndrome coronavirus 2 (SARS-CoV-2). Since then, it began to spread rapidly in Hubei Province, throughout China and abroad areas, which has turned into a worldwide public health emergency and reached pandemic status. ${ }^{1}$ By the time of November 22, 2021, the number of reported cases has surpassed $256,480,022$ with over 5, 145, 002 deaths worldwide. ${ }^{2}$

SARS-CoV-2 is extremely infectious and spread primarily through respiratory droplets and close exposure. As patients possibly infected with SARS-CoV-2 need to attend hospital, the incidence of nosocomial infection is conceivably high. ${ }^{3}$ Most patients infected with SARS-CoV-2 in hospitals were healthcare workers, among them nurses constituted the 


\section{Graphical Abstract}

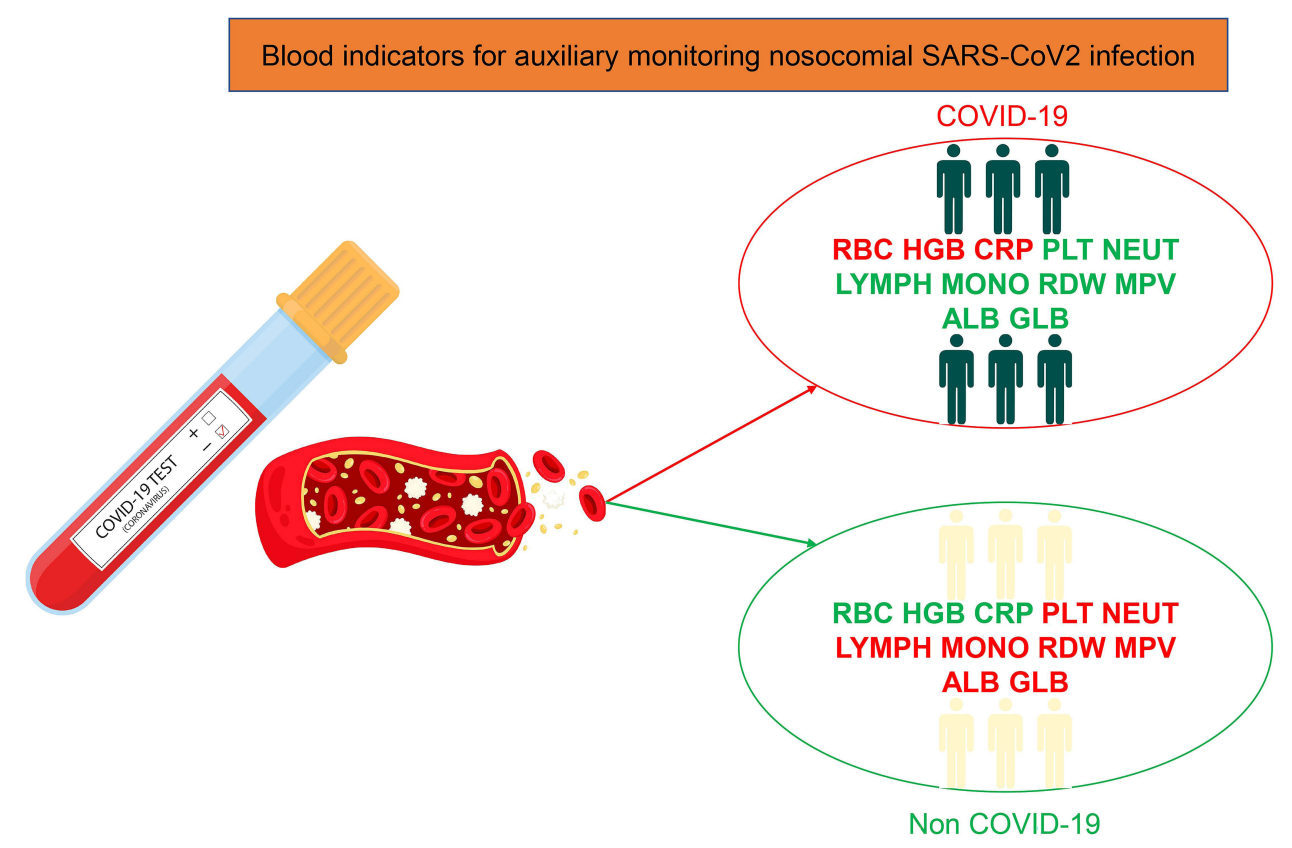

largest group, followed by doctors. Intensive surveillance of nosocomial SARS-CoV-2 infection in hospitalized patients is imperative to provide appropriate supportive care for patients, as well as prevent virus further transmission and avoid cross-contamination with non-infected individuals. So far, viral RNA detection by real-time quantitative PCR (RT-qPCR) and serological antibody test are most popular lab tests to confirm the diagnosis of SARS-CoV-2 infection. ${ }^{4}$ The positive detection rate of nucleic acid is only about $30 \%-50 \%$, mainly owing to many factors, such as sample types, different disease phages and techniques for specimens collecting and testing. ${ }^{5-7}$ Therefore, tests need to be repeated many times in suspected patients with initial negative results. ${ }^{8}$ In addition, the procedures for detection are laborious and timeconsuming. In contrast with RT-qPCR, serological antibodies test is beneficial with high reproducibility, short turnaround time and high biosecurity owing to the homogeneity of blood samples. ${ }^{9,10}$ Nevertheless, IgM and IgG antibodies titers are hinged largely on the time of antibody responses after infection, and they can be negative during the window periods. ${ }^{11}$ Despite recommendations to test hematological and biochemical indicators as part of the diagnostic workup for COVID-19, ${ }^{12,13}$ whether the routine laboratory tests could correctly distinguish cases of COVID-19 from non-infected inpatients is still doubtful. Our laboratory previously analyzed the hematological and biochemical parameters of 84 COVID-19 patients and 221 patients with community-acquired pneumonia (CAP), which indicated that most blood indexes of COVID-19 patients displayed obvious differences in contrast with CAP patients or healthy controls and some parameters could be predictive of COVID-19 diagnosis. ${ }^{14}$ Nevertheless, the individual indicator only had a fair discriminatory ability. To further evaluate the diagnostic value of the blood routine test, we constructed and validated a nomogram integrated eleven laboratory indexes to assist monitoring nosocomial SARS-CoV-2 infection in hospitalized patients.

\section{Patients and Methods}

\section{Data Collection}

Clinical data and laboratory indicators of 437 COVID-19 hospitalized cases and 420 negative inpatients between January 20th 2020 and May 25th 2020 were retrospectively collected from two clinical Centers: Zhongnan Hospital of Wuhan University and Taihe Hospital of Hubei University of Medicine. Clinical laboratory indicators of routine blood 
tests and blood biochemical parameters for the first time after admission were collected from routine clinical practice. All recruited COVID-19 patients were diagnosed based on the Guidelines for Diagnosis and Treatment of Novel Coronavirus Pneumonia (7th version). All control groups were negative inpatients for SARS-CoV-2 RNA and antibodies, and patients with hematologic diseases or radio-chemotherapy or critical illness were excluded. The study complied with the Declaration of Helsinki and was approved by the Ethics Committees of Zhongnan Hospital of Wuhan University (No. ZN2020015) and the Medical Ethics Committee of Taihe Hospital of Hubei University of Medicine (No. TH2020009). Informed written consent was waived due to the use of de-identified retrospective data.

\section{Laboratory Methods}

Clinical laboratory indicators consisted of white blood cell count (WBC), red blood cell count (RBC), hemoglobin (HGB), platelet (PLT), neutrophil count (NEUT), lymphocyte count (LYMPH), monocyte count (MONO), eosinophil count (EO), basophil count (BASO), hematocrit (HCT), mean corpuscular volume (MCV), mean corpuscular hemoglobin $(\mathrm{MCH})$, mean corpuscular hemoglobin concentration (MCHC), red blood cell distribution width coefficient variation (RDW-CV), mean platelet volume (MPV), albumin (ALB), globulin (GLB), alanine transaminase (ALT), aspartate aminotransferase (AST), blood urea nitrogen (BUN), creatinine (Cr), C-reactive protein (CRP), total bilirubin (TBIL), direct bilirubin (DBIL), and total bile acid (TBA). All hematology and biochemical indicators were analyzed by standard automatic analyzer using commercial kits according to the manufacturers' protocols. SARS-CoV-2 RNAs were extracted from throat swabs and tested using reverse transcription real-time quantitative PCR assays (RT-qPCR). SARS-CoV-2 antibodies were detected by colloidal gold kits or chemiluminescence techniques according to the manufacturer's instruction.

\section{Statistical Analysis}

Categorical variables were shown to be frequencies with proportions, and Chi-square test or Fisher's exact test were conducted to analyze the differences, as appropriate. Continuous variables were tested for normality distribution by the Kolmogorov-Smirnov test and were shown as mean \pm standard deviation (SD) or median with interquartile range (IQR) based on distributions. Independent $t$-test and Mann-Whitney $U$-test were tested for continuous variables with or without normal distributions, respectively. Two-sided $\mathrm{P}<0.05$ was considered to be statistically significant. All the data analysis was performed using R software (version 3.6.0).

Least absolute shrinkage and selection operator (LASSO) regression analysis was applied to identify relatively important features. The prediction model was constructed by logistic regression, support vector machine (SVM), decision tree classification and random forest (RF). The nomogram was built using rm packages, and the discriminative ability was assessed by the area under the receiver operating characteristic curve (ROC). ${ }^{15}$ The nomogram was validated internally in the development cohort and externally in the validation cohort. The calibration curves were utilized to compare the correlation between the predicted probabilities and the actual outcomes. ${ }^{16}$ Decision curve analysis (DCA) and clinical impact curve analysis (CICA) were implemented to assess clinical usability and benefits of the nomogram. ${ }^{17,18}$

\section{Results}

\section{Demographics and Clinical Characteristics of Participants in the Development and Validation Cohorts}

In the development cohort, 296 COVID-19 hospitalized cases and 305 negative inpatients were enrolled from Zhongnan Hospital of Wuhan University. A validation cohort consisting of 141 positive COVID-19 hospitalized cases and 115 negative inpatients was recruited from Taihe Hospital of Hubei University of Medicine. In two cohorts of our study, the distributions of age, gender and disease types were similar between cases and controls. Among the control group, both cohorts consisted of about $20 \%$ patients with community-acquired pneumonia, including bacterial pneumonia, fungal pneumonia, pneumocystosis or chlamydia pneumonia. In the development cohort, COVID-19 patients had significantly higher value for RBC, HGB, HCT, MCH, MCHC, ALT, AST, Cr and lower value for WBC, PLT, NEUT, LYMPH, MONO, EO, BASO, RDW, MPV, GLB and BUN than controls. There were no significant differences in MCV, ALB, CRP, TBIL, DBIL and TBA between cases and controls (Table 1). In the validation cohort, COVID-19 patients show 
Table I Demographics and Clinical Characteristics of Participants in the Development and Validation Cohorts

\begin{tabular}{|c|c|c|c|c|c|c|}
\hline \multirow[t]{2}{*}{ Variables } & \multicolumn{3}{|c|}{ Development Cohort } & \multicolumn{3}{|c|}{ Validation Cohort } \\
\hline & Case $(n=296)$ & Control $(n=305)$ & $\mathbf{P}$ & Case $(n=|4|)$ & Control $(n=115)$ & $\mathbf{P}$ \\
\hline Age (year) & $55(40.25,64)$ & $54(40,63)$ & 0.656 & $42(32,57)$ & $47(37,53)$ & 0.411 \\
\hline Gender & & & 0.082 & & & 0.932 \\
\hline Male & I 44 (48.6\%) & 170 (55.7\%) & & 78 (55.3\%) & $63(54.8 \%)$ & \\
\hline Female & $152(5 \mid .4 \%)$ & I 35 (44.3\%) & & $63(44.7 \%)$ & $52(45.2 \%)$ & \\
\hline Fever & & & 0.295 & & & 0.388 \\
\hline No & 208 (70.3\%) & $226(74.1 \%)$ & & $96(68.1 \%)$ & $84(73 \%)$ & \\
\hline Yes & $88(29.7 \%)$ & 79 (25.9\%) & & 45 (3I.9\%) & $31(27 \%)$ & \\
\hline Diarrhea & & & 0.09 & & & 0.409 \\
\hline No & 278 (93.9\%) & 275 (90.2\%) & & I 30 (92.2\%) & 109 (94.8\%) & \\
\hline Yes & $18(6.1 \%)$ & $30(9.8 \%)$ & & II (7.8\%) & $6(5.2 \%)$ & \\
\hline Basic disease & & & 0.438 & & & 0.561 \\
\hline No & 201 (67.9\%) & 198 (64.9\%) & & 92 (65.2\%) & 71 (6I.7\%) & \\
\hline Yes & 95 (32.1\%) & 107 (35.1\%) & & 49 (34.8\%) & 44 (38.3\%) & \\
\hline WBC (×10^9/L) & $4.99(3.8,6.32)$ & $6.94(5.38,8.87)$ & $<0.001$ & $5.93(4.48,7.4)$ & $7.21(5.2,10.2)$ & $<0.001$ \\
\hline $\operatorname{RBC}\left(\times 10^{\wedge} \mid 2 / L\right)$ & $4.06(3.75,4.4)$ & $3.83(3.27,4.3 \mathrm{I})$ & $<0.001$ & $4.64 \pm 0.64$ & $4.17 \pm 0.71$ & $<0.001$ \\
\hline HGB $(g / L)$ & $130(120.55,140.58)$ & I I8.2 (99, 132.45) & $<0.001$ & $146(132,159.5)$ & $131(115,149)$ & $<0.001$ \\
\hline PLT (× $\left.\times 10^{\wedge} 9 / L\right)$ & $187(143.25,228.75)$ & $212(156,260)$ & $<0.001$ & $201(165.5,230.5)$ & $219(157,278)$ & 0.097 \\
\hline NEUT (× & $3.13(2.27,4.15)$ & $4.62(3.42,6.6 \mathrm{I})$ & $<0.001$ & $3.93(2.82,5.28)$ & $5.01(3.24,7.93)$ & 0.001 \\
\hline LYMPH (×10^9/L) & I.I $(0.68,1.58)$ & $1.35(0.98,1.75)$ & $<0.001$ & $1.3(0.95,1.66)$ & $1.42(1.09,1.87)$ & 0.061 \\
\hline MONO (×10^9/L) & $0.42(0.32,0.53)$ & $0.5 \mathrm{I}(0.38,0.67)$ & $<0.001$ & $0.4(0.29,0.52)$ & $0.42(0.32,0.6)$ & 0.1 \\
\hline EO $\left(\times 10^{\wedge} 9 / L\right)$ & $0.02(0,0.12)$ & $0.09(0.03,0.17)$ & $<0.001$ & $0.03(0.01,0.08)$ & $0.05(0.02,0.12)$ & 0.006 \\
\hline BASO (×10^9/L) & $0.02(0.01,0.03)$ & $0.02(0.02,0.04)$ & $<0.001$ & $0.01(0.01,0.02)$ & $0.02(0.01,0.04)$ & $<0.001$ \\
\hline HCT (\%) & $37(34.5,40.15)$ & $35(29.85,39.45)$ & $<0.001$ & $42.6(39.4,46.9)$ & $38.7(34.3,44.1)$ & $<0.001$ \\
\hline $\mathrm{MCV}(\mathrm{fl})$ & $91.7(88.3,94.6)$ & $93(88.45,95.65)$ & 0.056 & $92.7(89.3,95.3)$ & $92.5(88.3,95.5)$ & 0.98 \\
\hline $\mathrm{MCH}(\mathrm{pg})$ & $32.1(30.83,33.2)$ & $31.2(29.8,32.25)$ & $<0.001$ & $31.3(30.25,32.25)$ & $31(29.8,32.6)$ & 0.783 \\
\hline $\mathrm{MCHC}(\mathrm{g} / \mathrm{L})$ & $348.55(343.03,355.1)$ & $335.5(329.45,341.75)$ & $<0.001$ & $337(332,343)$ & $336(328,342)$ & 0.38 \\
\hline RDW (\%) & $13(12.6,13.6)$ & $13.8(13.2,15.15)$ & $<0.001$ & $12.5(12.1,13)$ & $13(12.4,13.9)$ & $<0.001$ \\
\hline MPV (fl) & $8.6(7.9,9.6)$ & $9(8.2,9.8)$ & 0.002 & $9.3(8.7,10.2)$ & $10(8.9,10.9)$ & 0.002 \\
\hline ALB (g/L) & $39.15(35.5,42.28)$ & $37.7(31.95,43.85)$ & 0.134 & $44.8(41.95,46.7)$ & $39.5(36.8,42.2)$ & $<0.001$ \\
\hline GLB (g/L) & $28.6(26.33,31.08)$ & $30.7(27,34.7)$ & $<0.001$ & $28.6(26.35,31.25)$ & $28.2(24.9,32.6)$ & 0.953 \\
\hline ALT (U/L) & $24(16,42)$ & $18(1 I, 31)$ & $<0.001$ & $20(13.5,30)$ & $19(12,30)$ & 0.345 \\
\hline AST (U/L) & $25(19,38)$ & $21(16,30)$ & $<0.001$ & $21(18,28)$ & $21(14,34)$ & 0.696 \\
\hline
\end{tabular}


Table I (Continued).

\begin{tabular}{|c|c|c|c|c|c|c|}
\hline \multirow[t]{2}{*}{ Variables } & \multicolumn{3}{|c|}{ Development Cohort } & \multicolumn{3}{|c|}{ Validation Cohort } \\
\hline & Case $(n=296)$ & Control $(n=305)$ & $\mathbf{P}$ & Case $(n=141)$ & Control $(n=115)$ & $\mathbf{P}$ \\
\hline BUN (mmol/L) & $4.37(3.46,5.4 I)$ & $4.87(3.63,6.55)$ & 0.002 & $3.89(3.11,4.83)$ & $4.61(3.26,5.84)$ & 0.004 \\
\hline $\mathrm{Cr}$ (umol/L) & $63.55(53,74.2)$ & $60.3(48,73.65)$ & 0.045 & $83.4(71.8,94.1)$ & $76.4(67.5,89.9)$ & 0.015 \\
\hline CRP (mg/L) & $8.75(I .8,43.5 I)$ & $7.28(2.2,24.4 I)$ & 0.352 & $9.63(1.67,22.94)$ & $2.97(1.21,9.33)$ & $<0.001$ \\
\hline TBIL (umol/L) & II.35 $(8.8,14.98)$ & $10.8(7.7,17.35)$ & 0.344 & II $(8.5,15.3)$ & $12.8(9.5,18)$ & 0.014 \\
\hline DBIL (umol/L) & $2.2(1.7,3.28)$ & $2.3(1.5,3.55)$ & 0.895 & $6.1(4.1,9.95)$ & $6.5(4,11.4)$ & $0.74 I$ \\
\hline TBA (umol/L) & $3.35(1.9,5.4)$ & $3.3(1.7,5.65)$ & 0.941 & $1.8(1.5,2.6)$ & $2.8(1.6,5.4)$ & $<0.001$ \\
\hline
\end{tabular}

Abbreviations: WBC, white blood cell count; RBC, red blood cell count; HGB, hemoglobin; PLT, platelet; NEUT, neutrophil count; LYMPH, lymphocyte count; MONO, monocyte count; EO, eosinophil count; BASO, basophil count; HCT, hematocrit; MCV, mean corpuscular volume; MCH, mean corpuscular hemoglobin; MCHC, mean corpuscular hemoglobin concentration; RDW, red blood cell distribution width coefficient variation; MPV, mean platelet volume; ALB, albumin; GLB, globulin; ALT, alanine transaminase; AST, aspartate aminotransferase; BUN, blood urea nitrogen; Cr, creatinine; CRP, C-reactive protein; TBIL, total bilirubin; DBIL, direct bilirubin; TBA, total bile acid.

increased RBC, HGB, HCT, ALB, Cr and CRP, and decreased WBC, NEUT, EO, BASO, RDW, MPV, BUN, TBIL and TBA, compared with controls. The level of PLT, LYMPH, MONO, MCV, MCH, MCHC, GLB, ALT, AST and DBIL were comparable between COVID-19 patients and controls (Table 1).

\section{Features Selection and Machine Learning Model Evaluation}

Thirty variables measured at hospital admission were collected from each participant in the development cohort. After rejecting unrelated and superfluous variables, twenty variables were included in the LASSO regression analysis (Figure 1A). Using the LASSO regression filtration, eleven variables remain significant predictors of positive cases, including RBC, HGB, PLT, NEUT, LYMPH, MONO, RDW, MPV, ALB, GLB and CRP (Figure 1B). Then, we constructed prediction models using logistic regression, support vector machine (SVM), decision tree classification, random forest (RF), and the precision-recall curve (Figure 2) and the receiver operating characteristic curve (ROC) (Figure 3) were used to assess their accuracy. There were no obvious differences in the accuracy of these four models, and we chose the logistic regression model for further analysis due to its high interpretability.

\section{Construction, Evaluation and External Validation of a Nomogram}

The predictive nomogram based on the selected eleven variables from the development cohort was built to predict SARSCov-2 infection (Figure 1C). Each level of every variable was conferred a score on the scale of points. By summing up the scores of selected variables, a total score was available. The prediction possibility corresponding to this total score was used to predict SARS-CoV-2 infection in hospitalized patients. The calibration plot of the nomogram displayed that the predicted outcomes were in good concordance with the actual observations both in the development cohort and validation cohort (Figure 4A and B). The results of DCA and CICA indicated that the nomogram showed a better net benefit across a wide range of threshold probabilities and affected the outcome of patients (Figure 4C and D).

In the development cohort, the nomogram showed a high AUC 0.863 (95\% CI: 0.834-0.892) to identify COVID-19 patients from hospitalized patients, with a sensitivity of $80.41 \%$ and specificity of $77.38 \%$ (Figure $5 \mathrm{~A}$ ). In the validation cohort, AUC was 0.813 (95\% CI: $0.760-0.866$ ) for SARS-CoV-2 positive patients with a sensitivity of $82.98 \%$ and specificity of $70.43 \%$ (Figure $5 B$ ).

\section{Discussion}

Intensive surveillance of nosocomial SARS-CoV-2 infection in hospitalized patients is of great significance and may assist in providing patients with appropriate supportive care and preventing further virus transmission. In the current study, we identified eleven laboratory indicators, including RBC, HGB, PLT, NEUT, LYMPH, MONO, RDW, MPV, 


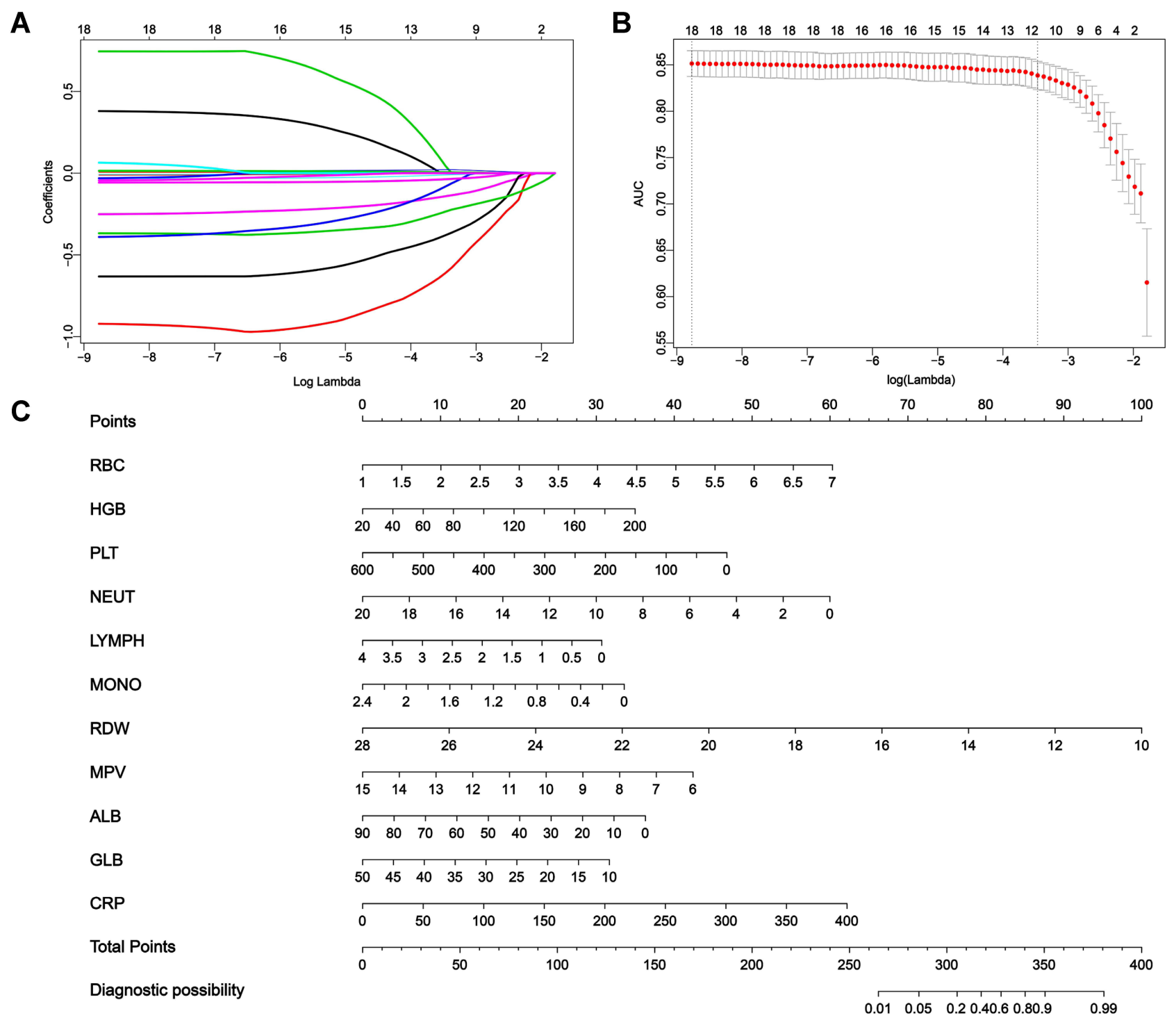

Figure I Construction of predictive nomogram in hospitalized patients with SARS-CoV-2 infection. (A) Least absolute shrinkage and selection operator (LASSO) coefficients profiles ( $y$-axis) of the twenty high dimensional predictors, which uses a LI penalty to shrink some regression coefficients to exactly zero. The upper $\mathrm{x}$-axis is the average numbers of predictors and the lower $x$-axis is the $\log (\lambda)$. (B) Fivefold cross-validation for tuning parameter selection in the LASSO model. The area under the receiver operating characteristic (AUC) with error bar is plotted against $\log (\lambda)$, where $\lambda$ is the tuning parameter. The dotted vertical lines are drawn at the optimal values by minimum criteria and the one standard error of the minimum criteria (Ise criteria). The upper $\mathbf{x}$-axis is the average numbers of predictors and the lower $\mathbf{x}$-axis is the log $(\lambda)$. To avoid overfitting, I se criteria $(\lambda=0.03 \mathrm{I})$ was selected. (C) Nomogram predicted SARS-CoV-2 infection of hospitalized patients with eleven laboratory indicators, including RBC, HGB, PLT, NEUT, LYMPH, MONO, RDW, MPV, ALB, GLB and CRP. Each level of every variable was conferred a score on the scale of points. By summing up the scores of selected variables, a total score was available. The prediction possibility corresponding to this total score was used to predict SARS-CoV-2 infection of hospitalized patients.

Abbreviations: RBC, red blood cell count; HGB, hemoglobin; PLT, platelet; NEUT, neutrophil count; LYMPH, lymphocyte count; MONO, monocyte count; RDW, red blood cell distribution width coefficient variation; MPV, mean platelet volume; ALB, albumin; GLB, globulin; CRP, C-reactive protein.

ALB, GLB and CRP, were associated with SARS-CoV-2 infection. Furthermore, we developed and validated an effectual nomogram integrated eleven features to predict SARS-CoV-2 infection in hospitalized patients with high sensitivity and specificity. The calibration plot showed a good consistency between the actual observations and the predicted outcomes both in the development cohort and validation cohort. DCA and CICA further implied that the nomogram displayed a better net benefit across a wide range of threshold probabilities and impacted the outcome of patients.

The standard approach for the etiological diagnosis of SARS-CoV-2 infection is nucleic acid detection by RT-qPCR. Although some patients were strongly epidemiologically correlated with SARS-CoV-2 exposure and presented symptoms of suspected viral pneumonia, the nucleic acid test of respiratory tract specimens did not show positive results generally 
A

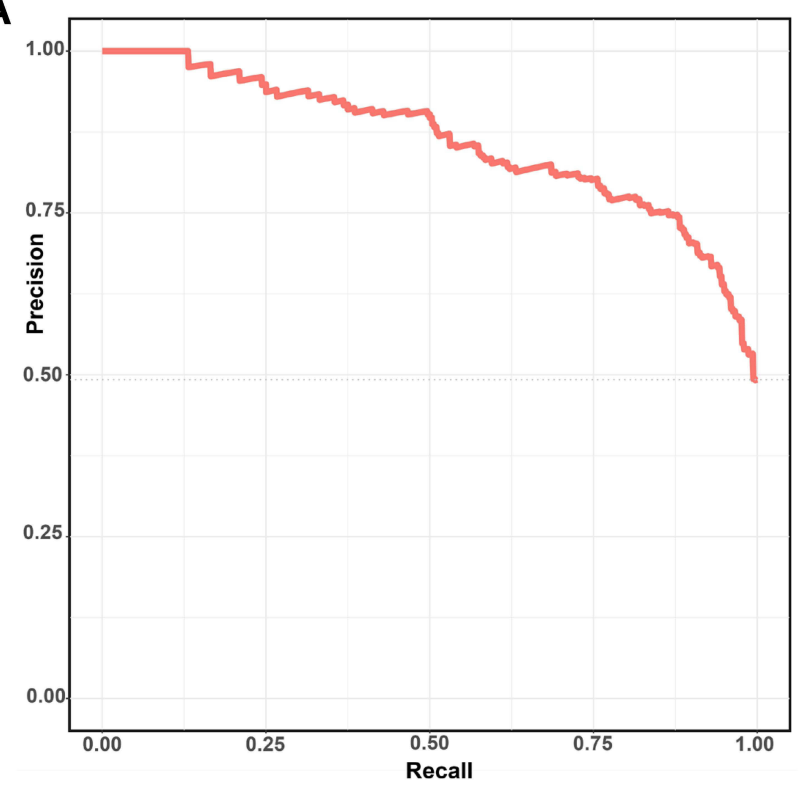

C

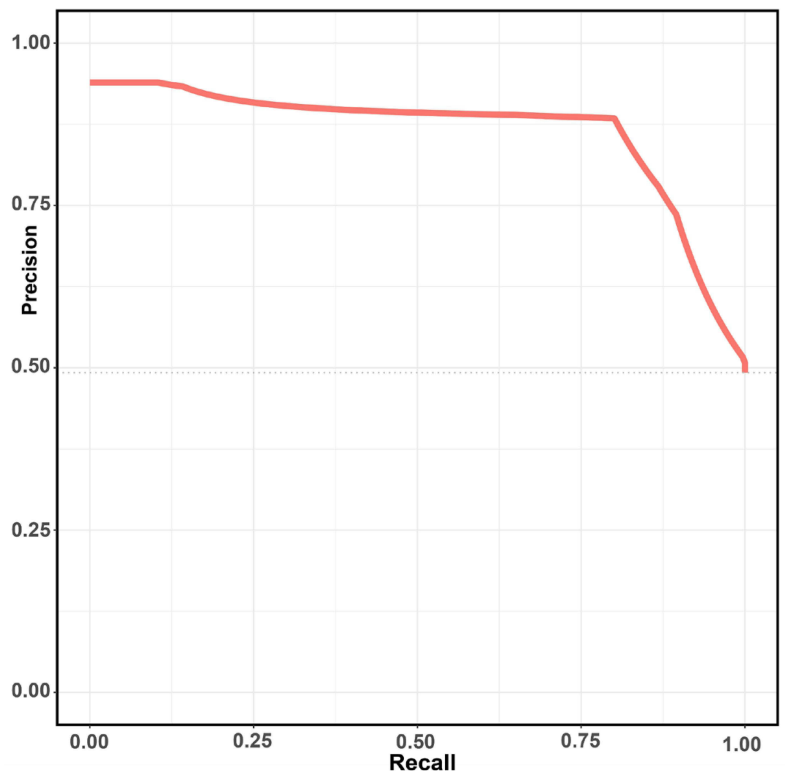

B

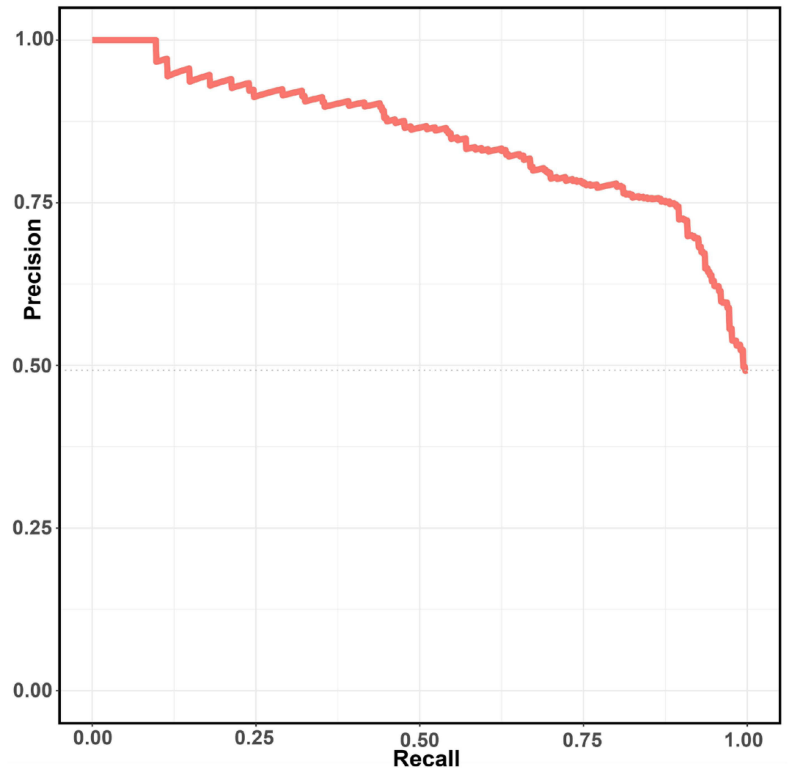

D

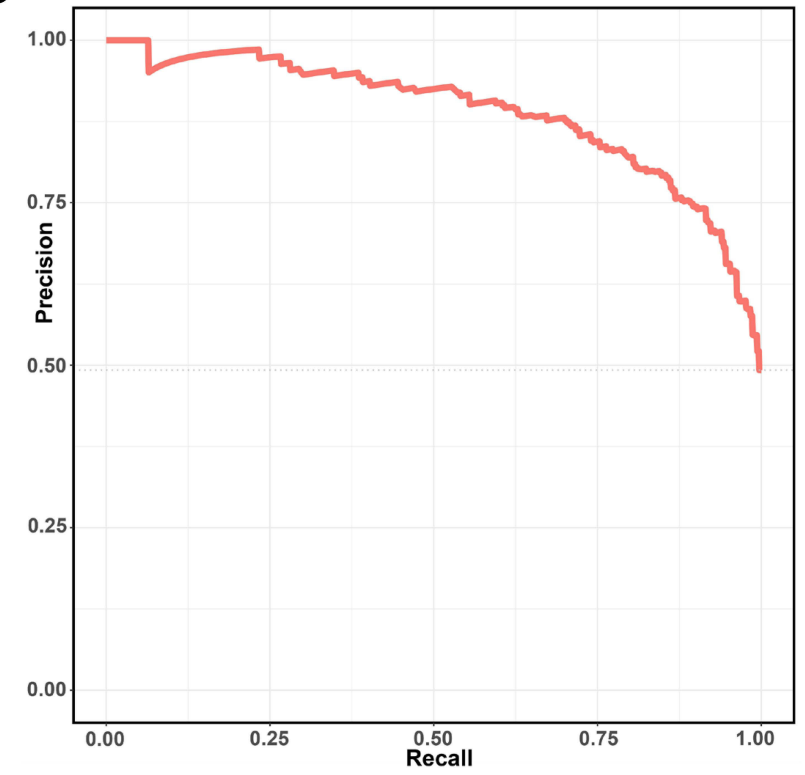

Figure 2 The precision-recall curve of four machine learning model. (A) Logistic regression. (B) Support vector machine (SVM). (C) Decision tree classification. (D) Random forest (RF).

until 5-6 days of symptom onset. Additionally, the sensitivity of nucleic acid test was also determined by different disease phages, sample types and techniques for collection, storage, transport and detection. ${ }^{5-7}$ Therefore, tests need to be repeated several times in patients with suspicion of being infected before confirming diagnosis, and they might not have enough priority to receive appropriate supportive care and isolation managements. ${ }^{19}$ In addition, the procedures of detection are laborious, and results may take at least several hours to be obtained. In contrast with RT-qPCR, Immunological detection of $\operatorname{IgM} / \operatorname{IgG}$ against SARS-CoV-2 is beneficial with short turn-around time, high biosecurity and high reproducibility owing to the homogeneity of blood samples. ${ }^{9,10}$ However, IgM and IgG antibody titers are hinged largely on the time of antibody responses after infection, and they can be negative during the window periods. ${ }^{11}$ Furthermore, serological antibody test cannot confirm SARS-CoV-2 existence and only provides evidence of recent infection. ${ }^{20}$ Our nomogram based on daily blood routine laboratory tests may be helpful to monitor nosocomial SARSCoV-2 infection and adopt timely isolation measures, while confirmatory results are not available. 

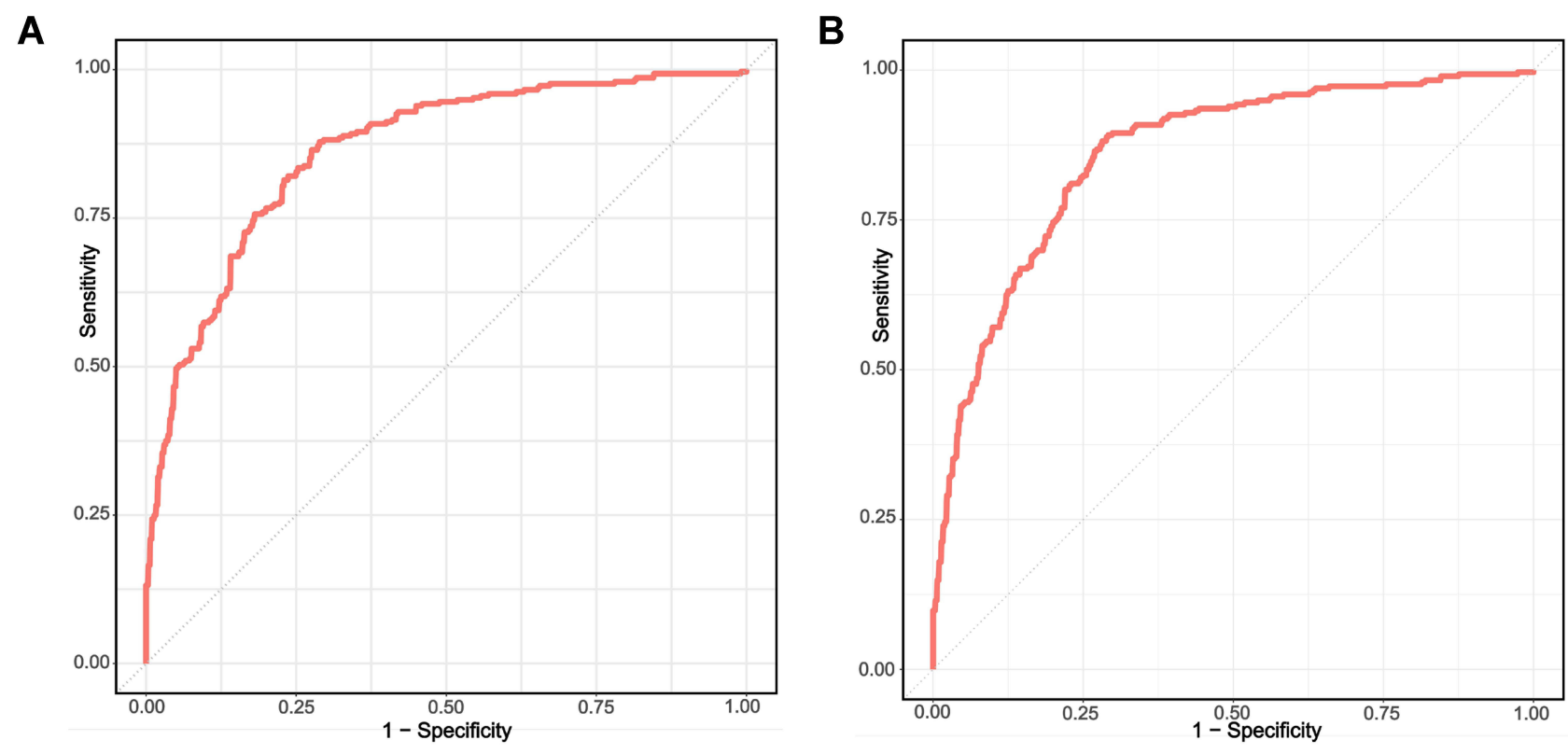

C

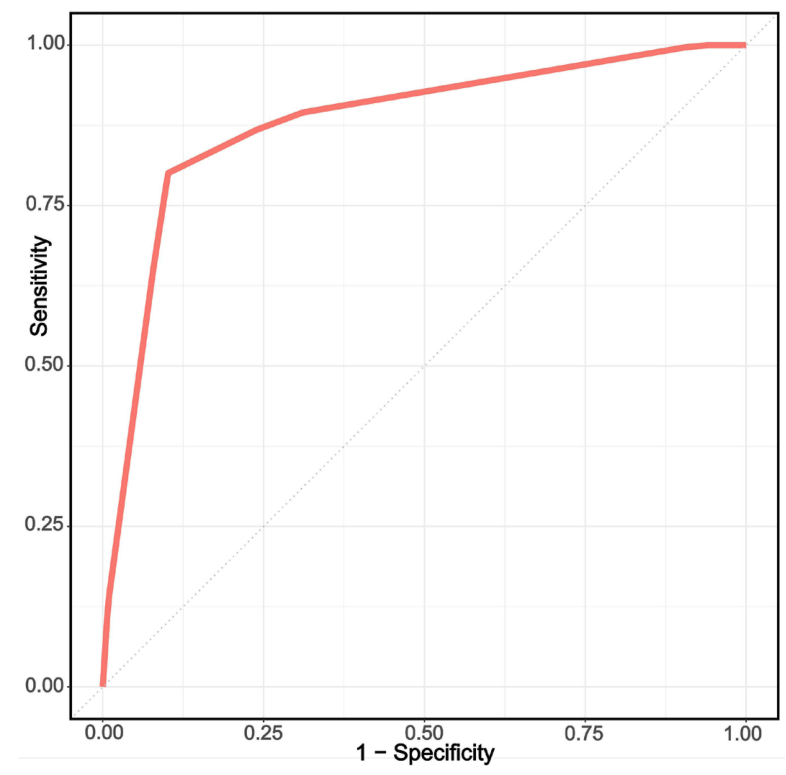

D

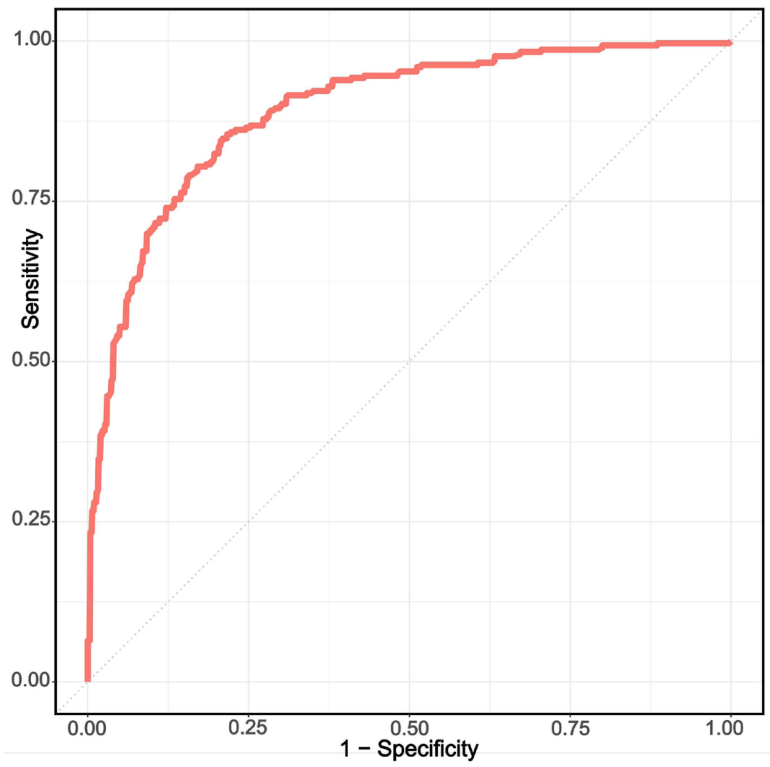

Figure 3 The ROC curve of four machine learning model. (A) Logistic regression. (B) Support vector machine (SVM). (C) Decision tree classification. (D) Random forest (RF).

Our nomogram integrated eleven easy-access laboratory indicators at hospital admission, including higher RBC, HGB, CRP and lower PLT, NEUT, LYMPH, MONO, RDW, MPV, ALB and GLB, similar results were obtained from the early investigation of the blood indexes of 84 COVID-19 patients and 221 CAP patients conducted by our laboratory, which signified that most blood parameters of COVID-19 patients showed noticeable discrepancies in contrast with CAP patients or healthy controls and some indexes could be predictive of COVID-19 diagnosis. ${ }^{14}$ Previous studies have already found that platelets, lymphocytes, neutrophil, monocyte, red blood cell distribution width coefficient variation, albumin and C-reactive protein are changeable at different degrees in COVID-19 patients. ${ }^{21-24}$ Hypoalbuminemia, lymphopenia and $\mathrm{CRP} \geq 4 \mathrm{mg} / \mathrm{dL}$ were reported as the predictive variables for pneumonia development against respiratory failure in patients with MERS-CoV infection. ${ }^{25}$ Liu et al indicated that lymphocytes count and albumin were negatively associated with the Murray scores, which were originally developed to evaluate the severity of acute lung injury, while $\mathrm{C}$ reactive protein level was positive with the Murray scores in COVID-19 patients. ${ }^{26}$ SARS-CoV-2 
A

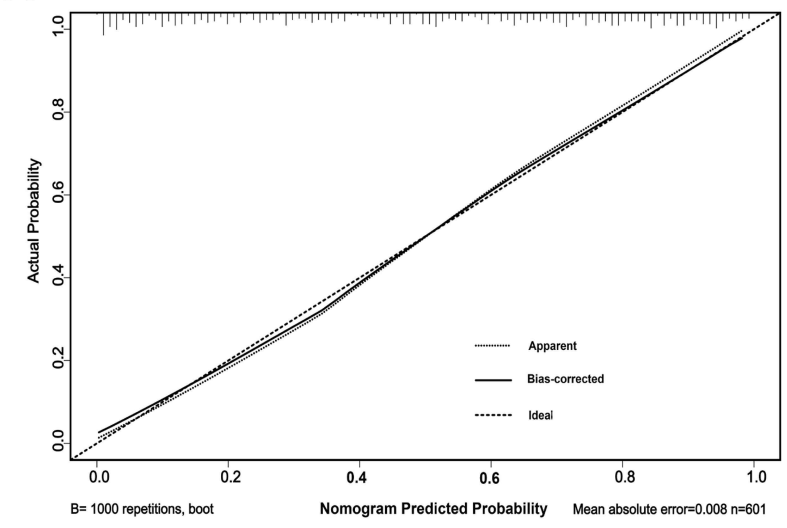

C

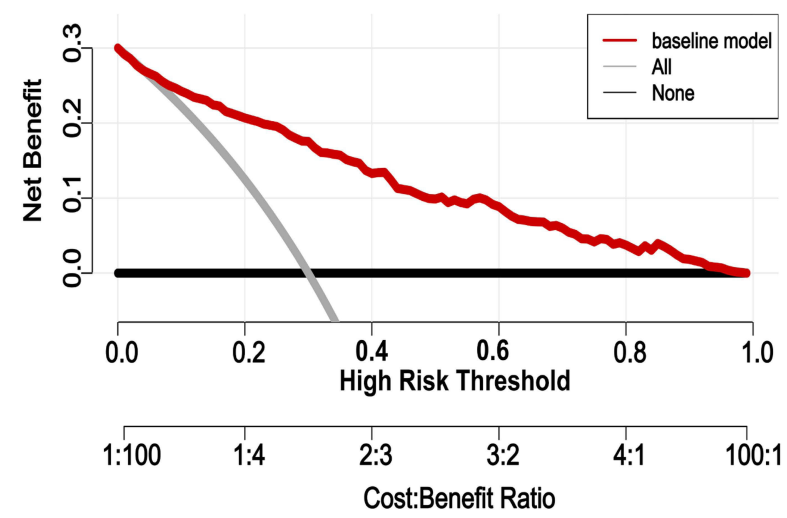

B

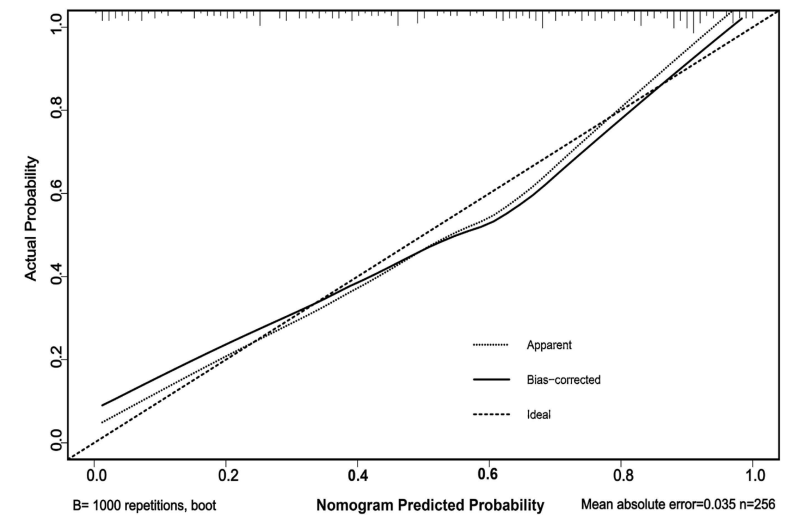

D
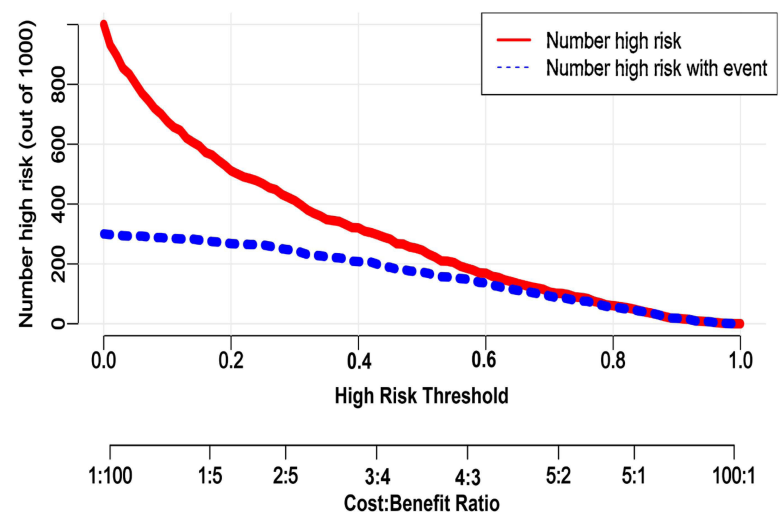

Figure 4 The calibration, DCA and CICA curves of the nomogram. (A) The calibration curve of the nomogram in the development cohort. (B) The calibration curve of the nomogram in the validation cohort. (C) The decision curve analysis (DCA) of the nomogram in the development cohort. The $y$-axis represents the net benefit and the $x$-axis represents the predicted probability of SARS-CoV-2 infection: a perfect prediction model (grey line), screen none (black line) and screen based on the nomogram (red line) (D) The clinical impact curve analysis (CICA) of the nomogram in the development cohort. The red line (number of high-risk individuals) is the number of people who are classified as positive by the model at each threshold probability, the blue line (number of high-risk individuals with event) indicates the number of true positives at each threshold probability.

A

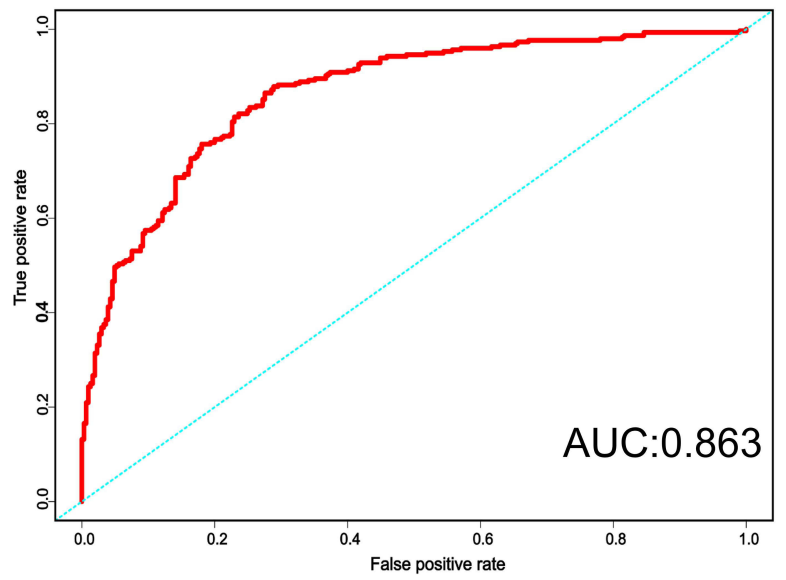

B

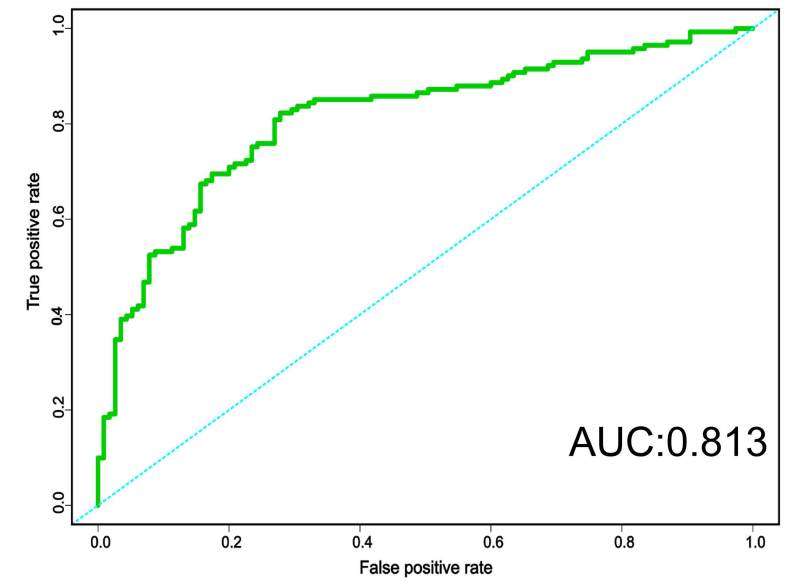

Figure 5 The ROC curve of the nomogram. (A) The ROC curve of the nomogram in the development cohort. (B) The ROC curve of the nomogram in the validation cohort. 
might work on lymphocytes, as does SARS-CoV, which elicits a cytokine storm and contributes to a series of immune responses. ${ }^{27}$ The decline of T lymphocyte count in COVID-19 patients implied that SARS-CoV-2 depleted immune cells and restrained the cellular immune function. ${ }^{22,28}$ In our previous study, we found that elevated RDW was a valuable prognostic marker for severe COVID-19. ${ }^{24}$ However, this study showed a decreased RDW in early SARS-CoV-2 infected patients comparing with non-COVID-19 patients. Therefore, further detailed study should be conducted to elucidate how the SARS-CoV-2 induces the dynamic change of blood components and which blood tests are more suggestive for differential diagnosis.

Our study has some advantages. First, with daily blood routine tests available, we could track and monitor inpatients that may have developed COVID-19, allowing to take more effective isolation measures to prevent virus further transmission. Second, to ensure the reliability of the conclusion, our nomogram was constructed with a relatively large sample size and validated externally using other populations. The performance of our nomogram was effectual for clinical application.

Several potential limitations of our study should be acknowledged. First, this is a retrospective study, which may have some inherent biases. Second, it was estimated that about $60 \%$ of SARS-CoV-2 infections are asymptomatic cases, possibly transmitting the virus before displaying any symptoms. ${ }^{29,30}$ However, clinical data and laboratory indicators of our study were collected during hospital admission, with patients having already presented symptoms and signs of COVID-19. Due to the lack of data on asymptomatic patients, we doubted whether our nomogram is suitable for asymptomatic infected individuals. Finally, the cohorts for development and validation were entirely from Hubei, China, which could restrict the generalizability of the nomogram in other areas of China. Therefore, further validation studies from domestic and abroad areas should be completed.

\section{Conclusion}

In summary, we constructed and validated a nomogram that integrated eleven laboratory indexes to assist monitoring SARS-CoV-2 infection of hospitalized patients. Our nomogram is extremely informative for clinical practice, which will be helpful for preventing SARS-CoV-2 further transmission in hospital and avoiding nosocomial infection.

\section{Acknowledgments}

We thank Pedro Sampaio Amorim for his kind help in data analysis. Pedro Sampaio Amorim is a Brazilian researcher with more than 8 years of experience in Data Science, passionate about solving real-world challenges using data modeling and analysis.

\section{Funding}

This work was supported by the 2019-nCoV emergency project of Hubei University of Medicine (2020XGFYZR01) from Chunyan Peng.

\section{Disclosure}

The authors declare that they have no conflicts of interest.

\section{References}

1. Meo SA, Alhowikan AM, Al-Khlaiwi T, et al. Novel coronavirus 2019-nCoV: prevalence, biological and clinical characteristics comparison with SARS-CoV and MERS-CoV. Eur Rev Med Pharmacol Sci. 2020;24:2012-2019. doi:10.26355/eurrev_202002_20379

2. World Health Organization. Coronavirus disease (COVID-19) weekly epidemiological update; 2020. Available from: https://www.who.int/publica tions/m/item/weekly-operational-update-on-covid-19-23-november-2021. Accessed November 23, 2021.

3. Zhou Q, Gao YL, Wang XM, et al. Nosocomial infections among patients with COVID-19, SARS and MERS: a rapid review and meta-analysis. Ann Transl Med. 2020;8:629. doi:10.21037/atm-20-3324

4. National Health Commission of the People's Republic of China. The guidelines for diagnosis and treatment of novel coronavirus pneumonia (7th version); 2020. Available from: http://www.nhc.gov.cn/yzygj/s7653p/202003/46c9294a7dfe4cef80dc7f5912eb1989.shtml. Accessed March 4, 2020.

5. Chu DKW, Pan Y, Cheng SMS, et al. Molecular diagnosis of a novel coronavirus (2019-nCoV) causing an outbreak of pneumonia. Clin Chem. 2020;66:549-555. doi:10.1093/clinchem/hvaa029 
6. Corman VM, Landt O, Kaiser M, et al. Detection of 2019 novel coronavirus (2019-nCoV) by real-time RT-PCR. Euro Surveill. 2020;25:2000045. doi:10.2807/1560-7917.ES.2020.25.3.2000045

7. Lippi G, Simundic AM, Plebani M. Potential preanalytical and analytical vulnerabilities in the laboratory diagnosis of coronavirus disease 2019 (COVID-19). Clin Chem Lab Med. 2020;58:1070-1076. doi:10.1515/cclm-2020-0285

8. Arevalo-Rodriguez I, Buitrago-Garcia D, Simancas-Racines D, et al. False-negative results of initial RT-PCR assays for COVID-19: a systematic review. PLoS One. 2020;15:e0242958. doi:10.1371/journal.pone.0242958

9. Xiang F, Wang X, He X, et al. Antibody detection and dynamic characteristics in patients with COVID-19. Clin Infect Dis. 2020;71:1930-1934. doi:10.1093/cid/ciaa461

10. Zhao R, Li M, Song H, et al. Early detection of SARS-CoV-2 antibodies in COVID-19 patients as a serologic marker of infection. Clin Infect Dis. 2020;71:2066-2072. doi:10.1093/cid/ciaa523

11. Li G, Chen X, Xu A. Profile of specific antibodies to the SARS-associated coronavirus. N Engl J Med. 2003;349:508-509. doi:10.1056/ NEJM200307313490520

12. Huang CL, Wang YM, Li XW, et al. Clinical features of patients infected with 2019 novel coronavirus in Wuhan, China. Lancet. 2020; 395: 497506.

13. Jin YH, Cai L, Cheng ZS, et al. A rapid advice guideline for the diagnosis and treatment of 2019 novel coronavirus (2019-nCoV) infected pneumonia (standard version). Mil Med Res. 2020; 7: 4.

14. Pan YB, Ye GM, Zeng XT, et al. Can routine laboratory tests discriminate SARS-CoV-2-infected pneumonia from other causes of communityacquired pneumonia? Clin Transl Med. 2020; 10: 161-168.

15. Hanley JA, McNeil BJ. The meaning and use of the area under a receiver operating characteristic (ROC) curve. Radiology. 1982; 143 : 29-36.

16. Coutant C, Olivier C, Lambaudie E, et al. Comparison of models to predict nonsentinel lymph node status in breast cancer patients with metastatic sentinel lymph nodes: a prospective multicenter study. J Clin Oncol. 2009;27:2800-2808. doi:10.1200/JCO.2008.19.7418

17. Vickers AJ, Elkin EB. Decision curve analysis: a novel method for evaluating prediction models. Med Decis Making. 2006;26:565-574. doi:10.1177/0272989X06295361

18. Zhou ZR, Wang WW, Li Y, et al. In-depth mining of clinical data: the construction of clinical prediction model with R. Ann Transl Med. 2019;7:796. doi:10.21037/atm.2019.08.63

19. Wang M, Wu Q, Xu W, et al. Clinical diagnosis of 8274 samples with 2019-novel coronavirus in Wuhan. MedRxiv. 2020;18. doi:10.1101/ 2020.02.12.20022327

20. Li Z, Yi Y, Luo X, et al. Development and clinical application of a rapid IgM-IgG combined antibody test for SARS-CoV-2 infection diagnosis. J Med Virol. 2020;92:1518-1524. doi:10.1002/jmv.25727

21. Cheng Z, Lu Y, Cao Q, et al. Clinical features and chest CT manifestations of coronavirus disease 2019 (COVID-19) in a single-center study in Shanghai, China. Am J Roentgenol. 2020;215:121-126. doi:10.2214/AJR.20.22959

22. Qin C, Zhou L, Hu Z, et al. Dysregulation of immune response in patients with COVID-19 in Wuhan, China. Clin Infect Dis. 2020;71:762-768. doi:10.1093/cid/ciaa248

23. Lu G, Wang J. Dynamic changes in routine blood parameters of a severe COVID-19 case. Clin Chim Acta. 2020;508:98-102. doi:10.1016/j. cca.2020.04.034

24. Gong J, Ou J, Qiu X, et al. A tool to early predict severe corona virus disease 2019 (COVID-19): a multicenter study using the risk nomogram in Wuhan and Guangdong, China. Clin Infect Dis. 2020;71:833-840. doi:10.1093/cid/ciaa443

25. Ko JH, Park GE, Lee JY, et al. Predictive factors for pneumonia development and progression to respiratory failure in MERS-CoV infected patients. J Infect. 2016;73:468-475. doi:10.1016/j.jinf.2016.08.005

26. Liu Y, Yang Y, Zhang C, et al. Clinical and biochemical indexes from 2019-nCoV infected patients linked to viral loads and lung injury. Sci China Life Sci. 2020;63:364-374. doi:10.1007/s11427-020-1643-8

27. Channappanavar R, Perlman S. Pathogenic human coronavirus infections: causes and consequences of cytokine storm and immunopathology. Semin Immunopathol. 2017;39:529-539. doi:10.1007/s00281-017-0629-x

28. Liu WJ, Zhao M, Liu K, et al. T-cell immunity of SARS-CoV: implications for vaccine development against MERS-CoV. Antiviral Res. 2017;137:82-92. doi:10.1016/j.antiviral.2016.11.006

29. Qiu J. Covert coronavirus infections could be seeding new outbreaks. Nature. 2020. doi:10.1038/d41586-020-00822-x

30. Zou L, Ruan F, Huang M, et al. SARS-CoV-2 viral load in upper respiratory specimens of infected patients. $N$ Engl J Med. 2020;382:1177-1179. doi:10.1056/NEJMc2001737

Journal of Inflammation Research

Dovepress

\section{Publish your work in this journal}

The Journal of Inflammation Research is an international, peer-reviewed open-access journal that welcomes laboratory and clinical findings on the molecular basis, cell biology and pharmacology of inflammation including original research, reviews, symposium reports, hypothesis formation and commentaries on: acute/chronic inflammation; mediators of inflammation; cellular processes; molecular mechanisms; pharmacology and novel anti-inflammatory drugs; clinical conditions involving inflammation. The manuscript management system is completely online and includes a very quick and fair peer-review system. Visit http://www.dovepress.com/testimonials.php to read real quotes from published authors.

Submit your manuscript here: https://www.dovepress.com/journal-of-inflammation-research-journal 